Neurol Med Chir (Tokyo) 50, $220 \sim 223,2010$

\title{
Massive Intracranial Hemorrhage Associated With Pleomorphic Xanthoastrocytoma -Case Report-
}

\author{
Gakushi YoshiKaWA, Shunsuke KAWAMOTO, \\ Kyoko YAKOU, and Kazuo TSUTSUMI \\ Department of Neurosurgery, Showa General Hospital, Kodaira, Tokyo
}

\begin{abstract}
A 60-year-old woman with a history of intermittent headaches and frequent seizures for 30 years presented with a massive intracranial hematoma in the left medial temporal lobe with thick subarachnoid hemorrhage. She had been treated with anticonvulsant medication under a diagnosis of left mesial temporal sclerosis based on magnetic resonance imaging findings. Cerebral angiography on admission revealed occlusion of the $\mathrm{P}_{2}$ segment of the left posterior cerebral artery (PCA) and extravasation of contrast medium during the procedure. The patient underwent left temporal lobectomy including the lesioned mesial temporal cortex, and the ruptured $P_{2}$ segment of the PCA was removed as well. The operative finding of the ruptured aneurysm was pseudoaneurysm. Histological examination of the resected PCA segment demonstrated a pleomorphic xanthoastrocytoma invading the outer wall of the PCA. Presumably the bleeding was caused by the rupture of a pseudoaneurysm secondary to leptomeningeal involvement of this typically benign tumor.
\end{abstract}

Key words: intracranial hemorrhage, leptomeningeal involvement, pleomorphic xanthoastrocytoma, subarachnoid hemorrhage, pseudoaneurysm

\footnotetext{
Received April 9, 2009; Accepted August 3, 2009
} 


\section{Introduction}

Pleomorphic xanthoastrocytoma is a rare subtype of glial neoplasm, which was first described as a distinct form of supratentorial astrocytoma in $1979 .{ }^{8}$ ) The tumor is typically located in the superficial temporal cortex, and the most common clinical presentation is seizures, although headache is also common in young patients. In contrast to the pleomorphic cytology, the tumor shows benign biological behavior. ${ }^{3,8)}$ Hemorrhage associated with low-grade astrocytoma is unusual, with few reports of hemorrhage associated with pleomorphic xanthoastrocytoma. ${ }^{11,22,23)}$ Leptomeningeal involvement is a frequent finding in pleomorphic xanthoastrocytoma, but arterial invasion is unknown.

We report an unusual case of intracranial hemorrhage supposedly due to rupture of a pseudoaneurysm secondary to leptomeningeal involvement by pre-existing pleomorphic xanthoastrocytoma in the adjacent area.

\section{Case Report}

A 60-year-old-woman presented with sudden onset of coma and right hemiparesis. She had a history of intermittent headache and frequent seizures for 30 years, and had been treated with anticonvulsants by a neurologist at another hospital under a diagnosis of left mesial temporal sclerosis based on magnetic resonance imaging (Fig. 1), which was obtained 3 years before the onset. She had no history of hypertension or trauma, no sign of infection, and was afebrile on admission. Computed tomography on admission showed a massive hematoma compressing the midbrain in the left ambient cistern and partly extending to the left thalamus accompanied by diffuse thick subarachnoid hemorrhage (Fig. 2). Cerebral angiography demonstrated occlusion of the posterior cerebral artery (PCA) at the $\mathrm{P}_{2}$ segment and subsequent extravasation of the contrast material (Fig. 3). The angiographical findings were consistent with rupture of the $\mathrm{P}_{2}$ segment of the PCA. Since her left pupil was dilated at the time of the extravasation, the patient was directly taken to surgery.

Emergent decompressive craniectomy was performed. The hematoma was evacuated, and temporal lobectomy was performed to alleviate uncal herniation. During the procedure, troublesome re-bleeding from the friable wall of the PCA was encountered, as likely with a pseudoaneurysm. Therefore, the proximal and distal ends of the $\mathrm{P}_{2}$ segment of PCA were obliterated with clips, but unfortunately a specimen of the most friable segment of the PCA, which was supposed to be the rupture site, could not be obtained because of the technical damage caused by the hemostatic procedure. Both the medial temporal segment and the residual $\mathrm{P}_{2}$ segment of the PCA were resected.

Histological examination showed a glial tumor with marked pleomorphism of the tumor cells, including spindle cells and 'bizarre' giant cells with foamy cytoplasm. Eosinophilic granular bodies were seen in some foci (Fig. 4A). Reticulin staining showed a fine network of reticulin surrounding the tumor cells. Glial fibrillary acidic protein (GFAP) staining was positive in most parts of the tumor,

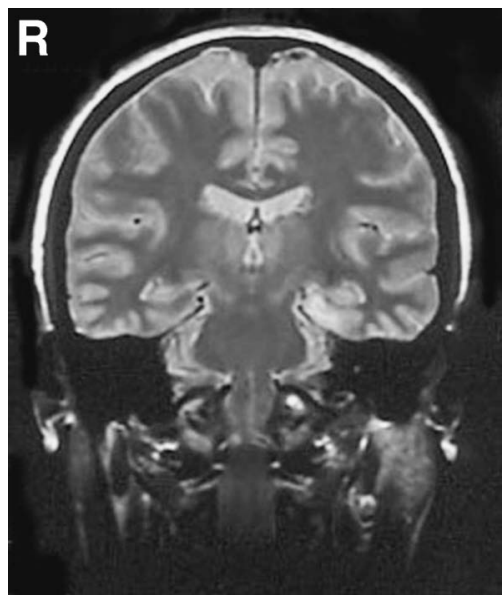

Fig. 1 Coronal $\mathrm{T}_{2}$-weighted magnetic resonance image obtained 3 years before onset showing high intensity in the left parahippocampal gyrus with no enhancement.
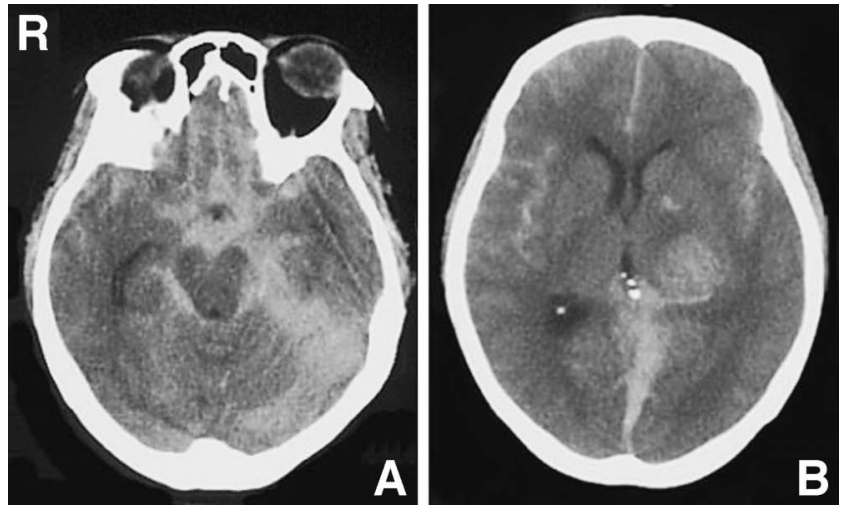

Fig. 2 (A) Computed tomography scan at the onset demonstrating massive hematoma in the left ambient cistern and thick subarachnoid hemorrhage. (B) Computed tomography scan demonstrating intraaxial hematoma in left thalamus.
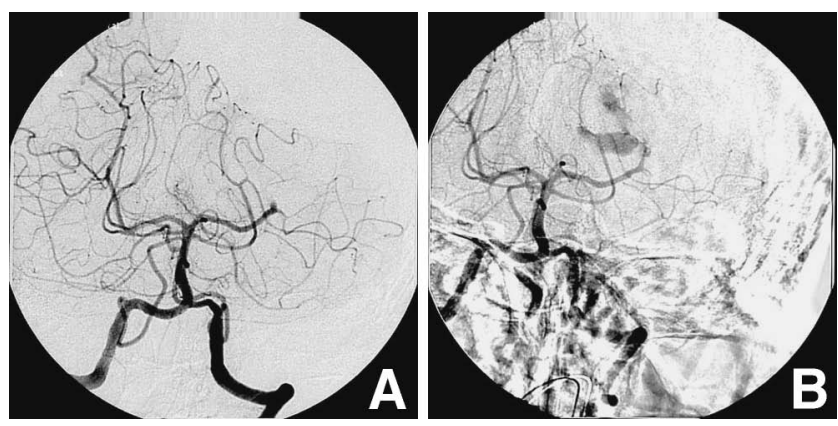

Fig. 3 (A) Left vertebral angiogram (anteroposterior view) showing occlusion of the posterior cerebral artery. (B) Subsequent left vertebral angiogram (anteroposterior view) showing extravasation of contrast medium. 


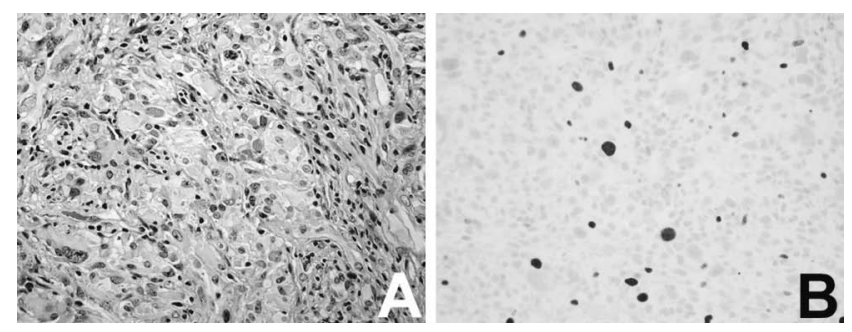

Fig. 4 (A) Photomicrograph showing markedly pleomorphic cells with giant and bizarre nuclei, and eosinophilic granular bodies, but no foci of necrosis. Hematoxylin and eosin stain, original magnification $\times 100$. (B) Photomicrograph showing MIB-1 index of less than $1 \%$. $\times 200$.

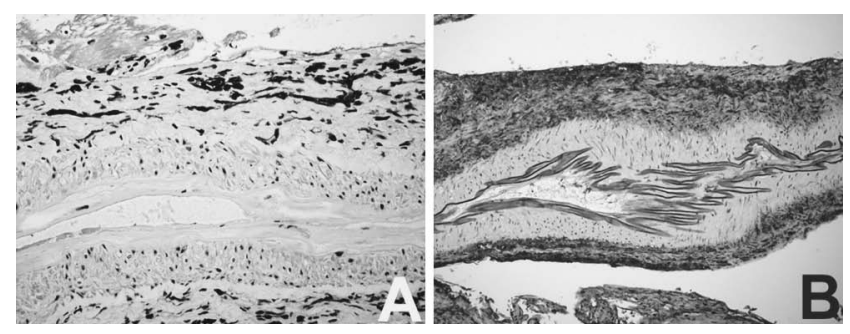

Fig. 5 (A) Photomicrograph of the posterior cerebral artery showing abnormal giant and bizarre cells around the perivascular space. Foci of invasion into the adventitial layer are seen. Hematoxylin and eosin stain, $\times 100$. (B) Photomicrograph of the abnormal giant cells around the perivascular area showing positive immunoreactivity to glial fibrillary acidic protein (GFAP) staining. The blue layer is the elastic laminae of the artery. GFAP and elastica stain, $\times 100$.

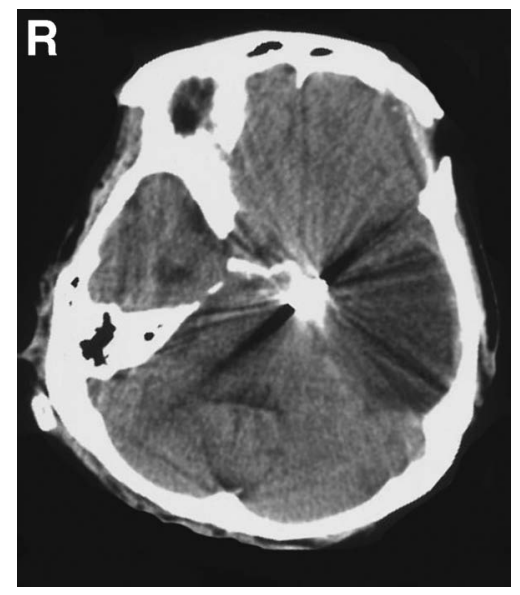

Fig. 6 Computed tomography scan demonstrating the defect of the left temporal lobe 3 months after the surgery.

though the MIB-1 index was less than 1\% (Fig. 4B). No necrotic foci were identified. These findings were consistent with pleomorphic xanthoastrocytoma. Areas adjacent to the ruptured PCA contained numerous bizarre and giant cells aggregating around the perivascular space and invading the adventitia of the vessel (Fig. 5A). Specimens obtained from the perivascular areas were positive for GFAP (Fig. 5B).

The patient underwent cranioplasty for the skull defect, and ventriculo-peritoneal shunting for hydrocephalus, but she remained bed-ridden due to the initial brain damage including the left thalamus and left cerebral infarction caused by subsequent vasospasm (Fig. 6).

\section{Discussion}

Bleeding due to intracranial neoplasms is relatively uncommon and occurs in only $1.5 \%$ of cases. ${ }^{12)}$ Intracranial hematoma is more frequent in glioblastoma/anaplastic gliomas and meningiomas than in other tumors. The incidence of bleeding from gliomas is approximately $5 \%, 9,13$ ) and is especially more common in high-grade gliomas such as glioblastoma/anaplastic astrocytoma, which shows tumor necrosis or endothelial proliferation, ${ }^{7,16}$ ) whereas low-grade astrocytomas comprise less than $1 \%$ of all tumors associated with hemorrhage. ${ }^{10)}$

Only a few cases of hemorrhage associated with lowgrade astrocytomas have been reported. ${ }^{2,4,5,14,17,20)}$ Possible causes of intratumoral hemorrhage include endothelial proliferation with vascular obliteration, vessel compression and/or distortion due to rapid tumor growth, vascular necrosis, abnormal structure of tumor capillaries such as retiform capillaries, and invasion of vessel walls by the tumor. ${ }^{7,13,16,20)}$ However, which histological features and what mechanisms actually cause such bleeding remain unclear. ${ }^{4)}$

In the first reported case of intracerebral and subarachnoid bleeding from a pleomorphic xanthoastrocytoma, temporal lobe and adjacent meningeal involvement by the neoplasm was proposed to have eventually produced erosion of an artery on the surface of the brain, but the cause of bleeding was not identified by imaging such as angiography. ${ }^{11)}$ Two other case reports described pleomorphic xanthoastrocytoma presenting with intratumoral bleeding. ${ }^{22,23)}$ An unknown microscopic vascular anomaly within the tumor parenchyma was proposed to induce massive bleeding, whereas leptomeningeal invasion was unlikely because of the absence of subarachnoid hemorrhage.

Our present case is the fourth hemorrhagic presentation of pleomorphic xanthoastrocytoma. Cerebral angiography revealed occlusion of the PCA and subsequent extravasation in areas adjacent to the hematoma. Operative findings included the very friable wall of the PCA that resulted in troublesome intraoperative re-bleeding, which was consistent with a pseudoaneurysm. She had no history of hypertension, trauma, or infectious event. Histological examination revealed that typical pleomorphic xanthoastrocytoma had surrounded the PCA and invaded its outer wall, although no specimen of the ruptured site of the PCA was obtained because of damage caused by the hemostatic procedure during the operation. A previous case of anaplastic pleomorphic xanthoastrocytoma with tumor bleeding had MIB-1 index of $9.8 \%$ indicating that malignant transformation might induce tumoral hemorrhage. ${ }^{1)}$ 
In contrast, our case of bleeding pleomorphic xanthoastrocytoma had MIB-1 index of less than 1\% and no foci of necrosis, so malignant transformation was unlikely as the cause of tumor bleeding.

The mechanisms of intracerebral hemorrhage from brain tumor are complex and involve multiple factors, but intracerebral hemorrhage from the neoplastic cerebral aneurysm is rare ${ }^{6,15)}$ Massive intracerebral hematoma induced by a neoplastic aneurysm caused by metastasis of lung carcinoma may have originated from a tumor cell migrating from the original site invading the wall of the peripheral artery, resulting the formation of an aneurysmal dilatation. On the other hand, glioblastoma with intratumoral bleeding was caused by the feeding artery pedicle aneurysm, which was presumably be formed by hemodynamic stress in the arteriovenous shunting associated with high-grade glioma such as glioblastoma. Unfortunately we were not able to detect tumoral invasion penetrating the arterial wall because of tissue damage, but we could show the pre-existing brain tumor and the resultant hemorrhage from the ruptured aneurysm which was directly attached to the tumor. Moreover, we demonstrated GFAP-positive tumor cells surrounding the outer wall of the posterior cerebral artery. Therefore, we speculate that the direct mural invasion of the tumor most likely caused formation of the pseudoaneurysm and subsequent rupture of the PCA, which led to the intraparenchymal and subarachnoid hemorrhage. Pleomorphic xanthoastrocytoma is well known for the presence of prominent basal lamina surrounding the tumor cells, which, in addition to its tendency to involve the leptomeninges, suggests subpial astrocytes as the possible origin of this rare tumor. ${ }^{8,18,19,21)}$ Invasion of vessel walls has not been recognized as a clinicopathological feature of pleomorphic xanthoastrocytoma, presumably because this tumor mostly involves the cerebral cortex and is usually not located in the proximity of major vessels. The present case is an important example of this interesting phenomenon, which may be helpful in the management of pleomorphic xanthoastrocytoma.

\section{References}

1) Asano K, Miyamoto S, Kubo O, Kikkukawa T, Yagihashi A, Ohkuma H: A case of anaplastic pleomorphic xanthoastrocytoma presenting with tumor bleeding and cerebrospinal fluid dissemination. Brain Tumor Pathol 23: 55-63, 2006

2) Devi BI, Shukla D, Bhat D, Santosh V: Hypothalamic tumour with haemorrhage. Childs Nerv Syst 17: 567-569, 2001

3) Giannini C, Scheithauer BW, Burger PC, Brat DJ, Wollan PC, Lach B, O'Neill BP: Pleomorphic xanthoastrocytoma: what do we really know about it? Cancer 85: 2033-2045, 1999

4) Golash A, Thorne J, West CG: Low grade pilocytic astrocytoma presenting as a spontaneous intracerebral haemorrhage in a child. Br J Neurosurg 12: 59-62, 1998

5) Gottfried ON, Fults DW, Townsend JJ, Couldwell WT: Spontaneous hemorrhage associated with a pilomyxoid astrocytoma. Case report. J Neurosurg 99: 416-420, 2003

6) Hashiguchi A, Morioka M, Ichimura H, Mimata C, Kuratsu $\mathrm{J}$ : Glioblastoma with an intratumoral feeding-artery aneurysm. Clin Neurol Neurosurg 109: 302-304, 2007

7) Hirano A, Matsui T: Vascular structures in brain tumors. Hum Pathol 6: 611-621, 1975

8) Kepes JJ, Rubinstein LJ, Eng LF: Pleomorphic xanthoastrocytoma: a distinctive meningocerebral glioma of young subjects with relatively favorable prognosis. A study of 12 cases. Cancer 44: 1839-1852, 1979

9) Kondziolka D, Bernstein M, Resch L, Tator CH, Fleming JF, Vanderlinden RG, Schutz H: Significance of hemorrhage into brain tumors: clinicopathological study. J Neurosurg 67: 852-857, 1987

10) Laurent JP, Bruce DA, Schut L: Hemorrhagic brain tumors in pediatric patients. Childs Brain 8: 263-270, 1981

11) Levy RA, Allen R, McKeever P: Pleomorphic xanthoastrocytoma presenting with massive intracranial hemorrhage. AJNR Am J Neuroradiol 17: 154-156, 1996

12) Licata B, Turazzi S: Bleeding cerebral neoplasms with symptomatic hematoma. J Neurosurg Sci 47: 201-210, 2003

13) Liwnicz BH, Wu SZ, Tew JM Jr: The relationship between the capillary structure and hemorrhage in gliomas. J Neurosurg 66: 536-541, 1987

14) Matsumoto K, Akagi K, Abekura M, Maeda $Y$, Kitagawa M, Ryujin $\mathrm{H}$, Iwasa $\mathrm{N}$ : Hypothalamic pilocytic astrocytoma presenting with intratumoral and subarachnoid hemorrhage. Neurol Med Chir (Tokyo) 37: 849-851, 1997

15) Nomura R, Yoshida D, Kim K, Kobayashi S, Teramoto A: Intracerebral hemorrhage caused by a neoplastic aneurysm from pleomorphic lung carcinoma. Neurol Med Chir (Tokyo) 49: 33-36, 2009

16) Scatliff JH, Radcliffe WB, Pittman HH, Park $\mathrm{CH}$ : Vascular structure of glioblastomas. Am J Roentgenol Radium Ther Nucl Med 105: 795-805, 1969

17) Sorenson EJ, Silbert PL, Benarroch EE, Jack CR, Parisi JE: Transient amnestic syndrome after spontaneous haemorrhage into a hypothalamic pilocytic astrocytoma. J Neurol Neurosurg Psychiatry 58: 761-763, 1995

18) Sugita $Y$, Kepes JJ, Shigemori M, Kuramoto S, Reifenberger G, Kiwit JC, Wechsler W: Pleomorphic xanthoastrocytoma with desmoplastic reaction: angiomatous variant. Report of two cases. Clin Neuropathol 9: 271-278, 1990

19) Tien RD, Cardenas CA, Rajagopalan S: Pleomorphic xanthoastrocytoma of the brain: MR findings in six patients. AJR Am J Roentgenol 159: 1287-1290, 1992

20) van Ouwerkerk WJ, Dirven CM: Hematoma in a low-grade medullary astrocytoma: report of an unusual case and literature review. Childs Nerv Syst 14: 742-746, 1998

21) Weldon-Linne CM, Victor TA, Groothuis DR, Vick NA: Pleomorphic xanthoastrocytoma. Ultrastructural and immunohistochemical study of a case with a rapidly fatal outcome following surgery. Cancer 52: 2055-2063, 1983

22) Wind JJ, Kerr PB, Sweet JA, Deshmukh VR: Pleomorphic xanthoastrocytoma presenting with life-threatening hemorrhage in a child. J Neurosurg Pediatr 3: 157-159, 2009

23) Yoshida D, Kogiku M, Noha M, Takahashi H, Teramoto A: A case of pleomorphic xanthoastrocytoma presenting with massive tumoral hemorrhage. J Neurooncol 71: 169-171, 2005

Address reprint requests to: Gakushi Yoshikawa, M.D., Department of Neurosurgery, Showa General Hospital, 2-450 Tenjin-cho, Kodaira, Tokyo 187-8510, Japan.

e-mail: gyoshika-tky@umin.ac.jp 